\title{
BASELINE FRAILTY EVALUATION IN DRUG DEVELOPMENT
}

\author{
F. CERRETA ${ }^{1}$, J. ANKRI ${ }^{2}$, D. BOWEN ${ }^{3}$, A. CHERUBINI ${ }^{4}$, A.J. CRUZ JENTOFT 5 , A. GUĐMUNDSSON 6 , \\ M. HABERKAMP ${ }^{7}$, P. JANSEN ${ }^{8}$, N. MARCHIONNI ${ }^{9}$, S. MORGAN $^{10}$, E. RÖNNEMAA $^{11}$, \\ M. PETROVIC ${ }^{12}$, A. PILOTTO ${ }^{13}$, M. ROSA ${ }^{14}$, H. WILDIERS ${ }^{15}$
}

\begin{abstract}
1. European Medicines Agency, London, United Kingdom; 2. UMR-S 1168, Université Versailles St-Quentin-en-Yvelines, France; 3 . European Medicines Agency, London, United Kingdom; 4. University of Perugia, Italy; 5. Hospital Universitario Ramon y Cajal, Spain; 6. National University Hospital of Iceland, Iceland; 7. Federal Institute of Drugs and Medical Devices, Germany; 8. Expertise centre Pharmacotherapy in Old Persons, the Netherlands; 9. University of Florence, Italy; 10. Medicines and Healthcare products Regulatory Agency, United Kingdom; 11. Medical Products Agency, Sweden; 12. Ghent University, Belgium; 13. Galliera Hospital, Genoa, Italy; 14. Faculdade de Medicina de Lisboa, Portugal; 15. University Hospitals Leuven, Belgium

Corresponding author: Francesca Cerreta; 7 Westferry Circus, Canary Wharf, London E14 4HB, United Kingdom; Phone: +44 (0)20 $74188400 ;$ Fax: +44 (0)20 74188613 ; email: Francesca.cerreta@ema.europa.eu
\end{abstract}

Older people are high consumers of medicines, but despite this they have often been excluded from clinical trials. Reasons for exclusion have frequently been poorly justifiable, and have included predefined arbitrary upper age limits, lists of different comorbidities, polypharmacy or physician/patient/ family preference. This selection bias is even more evident for the frail older adults. Consequently, the evidence base in this age group is often limited at the time of market authorisation. The benefit/risk analysis for the frail older adult may well be different from that of younger people, and a lack of data may result in inappropriate prescribing.

Frailty is a term used to identify older adults who have reduced resistance to stressors and are consequently at increased risk of poor clinical outcomes, such as incident disability, cognitive decline, falls, hospitalization, institutionalization, or increased mortality. Frail older persons are also vulnerable to clinically important adverse drug reactions. Hospital admissions related to medicines are especially seen in these patients and are often preventable1. Cross-sectional studies suggest that about $7 \%$ of persons older than 65 years are frail, and that the prevalence of frailty increases with age and may exceed $45 \%$ after age 85 .

Current regulatory guidance on clinical trials in the older population from the International Conference on Harmonisation (ICH E7) considers the following as important elements in the development of a new drug: recruitment of sufficient numbers of elderly patients in appropriate age ranges for pharmacokinetics (PK) and PK/pharmacodynamic (PD) analyses, the use of an age-appropriate measure of renal function, and awareness of and openness to testing covariates reflecting biological rather than chronological age. Older patients often exhibit enhanced PD sensitivity and thus exploration of the minimum effective dose is key to improving tolerability. The updated European Union Clinical Trials Regulation (536/2014) requires a justification for the gender and age allocation of subjects and, if a specific gender or age group is excluded from or underrepresented in the clinical trials, an explanation of the reasons and justification for these exclusion criteria. The ICH E7 Question and Answers advocates that it is very important to ensure, to the extent possible, that the population included in the clinical development program is representative of the target patient population. It is recognised that chronological age alone is a poor predictor of susceptibility to adverse health outcomes. In an effort to promote the use of an additional set of parameters to chronological age, the European Medicines Agency (EMA) Geriatric Expert Group (GEG), on behalf of the Committee for Medicinal Products for Human Use (CHMP) has developed a 'Points to Consider' document outlining the general principles that may be applied for the baseline categorisation of older patients enrolled in a clinical trial or other clinical investigation (e.g. registry) on the basis of their frailty status. In routine clinical practice, the GEG strongly recommends that frailty is not evaluated outside the framework of a multidimensional interdisciplinary comprehensive geriatric assessment.

The document will be available for public comment via the EMA public website (http://www.ema.europa.eu).

The following four aspects of frailty are considered with corresponding recommended instruments to categorise patients in these domains on the basis of their baseline frailty status at entry into the clinical trial. Parameters considered in the choice of instrument include validation status, predictive value, and ease of use, and represent expert opinion by consensus:

- Physical frailty: Short Physical Performance Battery (SPPB)

o The SPPB assesses lower-extremity function by measures of three separate tests, i.e. standing balance, walking speed, and ability to rise from a chair2,3. Should it not be practical to assess physical frailty by SPPB then gait speed is an alternative instrument, though not as well validated, nor as multifaceted as SPPB. In patients with lower limb disorders, there are no instruments available with validation comparable to SPPB but hand grip strength, upper arm circumference, or selected instruments used to assess sarcopenia would be alternative options.

- Cognitive dysfunction: Montreal Cognitive Assessment (MoCA)

o Cognitive dysfunction in the context of frailty is poorly studied compared to physical frailty and as such the most suitable instruments for assessment are less well 


\section{BASELINE FRAILTY EVALUATION IN DRUG DEVELOPMENT}

validated. A number of epidemiological studies have reported that frailty increases the risk of future cognitive decline and that cognitive impairment increases the risk of physical frailty suggesting that cognition and frailty interact mutually. Cognition is not only influenced by physical frailty but also by psychosocial parameters. Mini Mental State Examination (and its modification 3MS) is widely used in clinical trials but has copyright restrictions. MoCA is sensitive to mild cognitive impairment and includes domains not present in Mini Mental State Examination. It is a well-validated instrument in older subjects.

- Malnutrition: Mini-Nutritional Assessment - Short Form (MNA-SF)

o The European Society for Clinical Nutrition and Metabolism endorsed the 30-point Mini-Nutritional Assessment scale, a short form of which (the MNASF)4 is now widely used in clinical research and clinical practice. It is accurate to detect both potential and established under-nutrition. Although the MNA-SF should be considered standard, clinical trials requiring a more detailed nutritional assessment may consider using the full 30-item MNA instrument.

- Multi-morbidity: Cumulative Illness Rating Scale Geriatrics (CIRS-G)

o This scoring system measures the chronic medical illness («morbidity») burden while taking into consideration the severity of chronic diseases in 14 items representing individual body systems. It has been validated in older in-patients and outpatients, and in long-term care patients5. Criterion validity has been confirmed using autopsy as 'gold standard', and the instrument has good inter-rater and test-retest reliability. It predicts mortality, hospital readmission, prolonged hospital stay and nursing home admission.

This 'Points to Consider' document provides a menu of validated and therefore recommended instruments. Individual instruments (or combinations) to characterize the baseline frailty status may be selected for a clinical development program, according to the therapeutic area and the PD profile of the medicinal product under investigation. In the absence of specific pharmacodynamic parameters of interest but a desire to broadly characterize baseline frailty with one instrument, the determination of physical frailty status by SPPB testing is the preferred option as it has a strong correlation to adverse outcomes including mortality, nursing home admission and subsequent disability. This menu is not exhaustive and other validated instruments may be more suitable in specific circumstances. The development and validation of alternative / additional scales to better characterize specific patient populations is encouraged.

A standardized characterization of frailty is potentially useful for risk stratification and to improve the description of the characteristics of older populations involved in clinical trials or post-authorisation registries. If such frailty scales were to be routinely introduced to characterize baseline demographics of the population enrolled in a clinical trial for a drug with highly prevalent use in the older population, this might encourage active inclusion of frail patients thereby enhancing the assessment of the benefit/risk balance of the product in the target 'real world' population.

* The authors of the paper are members of the EMA Frailty Guidance Drafting Group.

\section{References}

1. Pirmohamed M, James S, Meakin S, et al. Adverse drug reactions as cause of admission to hospital: prospective analysis of 18820 patients. BMJ 2004;329:15-9.

2. Guralnik JM, Simonsick EM, Ferrucci L, et al. A short physical performance battery assessing lower extremity function: association with self-reported disability and prediction of mortality and nursing home admission. J Gerontol 1994;49:M85-94.

3. Guralnik JM, Ferrucci L, Simonsick EM, Salive ME, Wallace RB. Lowerextremity function in persons over the age of 70 years as a predictor of subsequent disability. N Engl J Med 1995;332:556-61.

4. Kaiser MJ, Bauer JM, Uter W, et al. Prospective validation of the modified mini nutritional assessment short-forms in the community, nursing home, and rehabilitation setting. J Am Geriatr Soc 2011;59:2124-8.

5. Salvi F, Miller MD, Grilli A, et al. A manual of guidelines to score the modified cumulative illness rating scale and its validation in acute hospitalized elderly patients. J Am Geriatr Soc 2008;56:1926-31. 